\title{
Specific expression and export of the Plasmodium falciparum Gametocyte EXported Protein-5 marks the gametocyte ring stage
}

\author{
Marta Tibúrcio ${ }^{1,3 \dagger}{ }^{\dagger}$, Matthew W. A. Dixon ${ }^{2 \dagger}$, Oliver Looker ${ }^{2}$, Sumera Younis Younis ${ }^{1,4}$, Leann Tilley $^{2 *}$ and Pietro Ala \\ no ${ }^{1 *}$
}

\begin{abstract}
Background: Plasmodium falciparum sexual development plays a fundamental role in the transmission and spread of malaria. The ability to generate gametocytes can be lost during culture in vitro, often associated with the loss of a subtelomeric region of chromosome 9 . Gametocytogenesis starts with erythrocyte invasion by a sexually committed merozoite, but the first available specific marker of sexual differentiation appears only from $24 \mathrm{~h}$ post invasion.

Methods: Specific antibodies and gene fusions were produced to study the timing of expression and the sub-cellular localization of the P. falciparum Gametocyte EXported Protein-5 (PfGEXP5), encoded in the subtelomeric region of chromosome 9. Expression patterns were examined in wild-type parasites and in parasite lines mutated in the Apetala2-G (AP2-G) transcription factor, governing a cascade of early sexual stage specific genes.

Results: PfGEXP5 is highly expressed in early sexual stages and it is actively exported to the infected erythrocyte cytoplasm from as early as $14 \mathrm{~h}$ post-invasion in haemozoin-free, ring stage-like parasites. The pattern of PfGEXP5 expression and export is similar in wild-type parasites and in independent AP2-G defective parasite lines unable to produce gametocytes.

Conclusions: PfGEXP5 represents the earliest post-invasion sexual stage marker described to date. This provides a tool that can be used to identify sexually committed ring stage parasites in natural infections. This early gametocyte marker would enable the identification and mapping of malaria transmission reservoirs in human populations and the study of gametocyte sequestration dynamics in infected individuals. The fact that regulation of PfGEXP5 does not depend on the AP2-G master regulator of parasite sexual development suggests that, after sexual commitment, differentiation progresses through multiple checkpoints in the early phase of gametocytogenesis.
\end{abstract}

Keywords: Plasmodium, Gametocyte, Exported protein, Chromosome 9, Commitment, Sexual differentiation, Early gametocyte marker

\section{Background}

Malaria is a vector-borne disease caused by the genus Plasmodium. It is responsible for an estimated 200

\footnotetext{
*Correspondence: Itilley@unimelb.edu.au; alano@iss.it

${ }^{\dagger}$ Marta Tibúrcio and Matthew W. A. Dixon contributed equally to this work

${ }^{1}$ Dipartimento di Malattie Infettive, Parassitarie e Immunomediate,

Istituto Superiore di Sanità, Viale Regina Elena 299, 00161 Rome, Italy

2 Department of Biochemistry and Molecular Biology, Bio21 Molecular

Science and Biotechnology Institute, The University of Melbourne,

Melbourne, VIC, Australia

Full list of author information is available at the end of the article
}

million clinical cases and approximately 580,000 deaths per year, mostly in sub-Saharan Africa [1], particularly affecting pregnant women and children. Of the five malaria species that infect humans, Plasmodium falciparum is the most virulent, responsible for $90 \%$ of disease cases [2].

Disease is associated with a $44-48 \mathrm{~h}$ cycle of asexual replication that takes place within the red blood cells (RBCs) of the human host, where P. falciparum matures through the ring, trophozoite and schizont stages. Clinical symptoms range from uncomplicated fevers to 
life-threatening cerebral and placental malaria [3]. During asexual cycling a portion of the parasite population commits to sexual differentiation (gametocytogenesis), thus ensuring parasite transfer to the transmission vector, an Anopheles mosquito [4].

Parasite sexual commitment occurs in the asexual generation preceding gametocytogenesis, where each individual schizont produces a progeny of merozoites that uniformly develop into either asexual parasites or sexual parasites of the same sex [5-7]. It is currently debated whether in natural infections a constant fraction of parasites converts to sexual development during each asexual cycle or whether commitment is an environment-sensitive, triggered event [8].

After invasion, a sexually committed parasite develops intra-erythrocytically over a period of ten to 12 days, progressing through five distinct stages of gametocyte development $[9,10]$. In the earliest stage of sexual development, the intra-erythrocytic parasite is morphologically indistinguishable from an asexual ring-stage parasite, and no specific markers are currently available to identify this 'sexual ring' stage. At $24-30 \mathrm{~h}$ post invasion (pi), about the time an asexual parasite enters the trophozoite stage, the early gametocyte (stage Ia) starts to produce the early stage-specific proteins Pfs16 and Pfg27 [11-15]. At about $48 \mathrm{~h}$ pi, ultrastructural features of the sexual parasite first become apparent (stage Ib to stage II), such as the assembly of a bilamellar membrane structure subtended by a network of subpellicular microtubules $[16,17]$.

In natural infections, stages Ib to IV gametocytes are sequestered in deep tissues, such as the bone marrow $[18,19]$. The only morphologically recognizable gametocyte stage that is visible in the peripheral circulation is stage V. Following ingestion by a mosquito, these mature gametocytes are responsive to the triggers that initiate gamete formation, the first step in transmission to the insect vector $[4,20]$. In contrast, the lack of specific markers for sexually committed parasites at the ring stage makes it impossible to answer the fundamental question of whether the earliest gametocyte stages (i.e., before $24 \mathrm{~h} \mathrm{pi}$ ) are formed, and remain, within the deep tissues or are freely circulating $[18,21]$. In addition, the lack of a sexual ring-stage diagnostic reagent makes it difficult to address key epidemiological questions relating to the dynamics of parasite transmission. For example, the identification of the human malaria reservoirs, such as long-term carriage of infective sub-microscopic gametocytes in asymptomatic individuals, would be facilitated by a ring stage marker [21]. Similarly, early detection of gametocytes would be important to evaluate the effects of different treatment regimens of symptomatic individuals on gametocyte carriage [22, 23].
Both of these questions have important implications for malaria control.

The ability of parasite lines and clones to generate gametocytes can be lost during culture in vitro. In $P$. falciparum, the gametocyteless clone F12 was derived from a gametocyte-producing parental 3D7 clone in a controlled 18-month asexual propagation experiment [24]. F12 parasites are unable to produce morphologically recognizable gametocytes, nor to express the stage I marker Pfg27 [24]. Exon sequencing of the genomes of F12 and of independently obtained gametocyteless parasites, such as GNP-A4, was used to identify the genetic defect [25]. Distinct null mutations in the coding sequence of a putative transcription factor of the P. falciparum Apetala2 family member, PfAP2-G, were shown to be associated with defective gametocyte production [25]. Gene disruption and down-regulation of the PfAP2-G expression functionally confirmed the role of this protein. PfAP2-G appears to be responsible for switching on the expression of a small set of early gametocyte proteins, which leads to the appearance of morphologically recognizable gametocytes [25].

The loss of gametocyte production has also been observed in parasite lines with different genetic backgrounds. For example the $\mathrm{C} 10$ parasite line, which is derived from isolate 1776 [26] produces no morphologically distinguishable gametocytes [24]. In this and in other lines of different geographical origin, the loss or dramatic reduction of gametocyte production is associated with subtelomeric deletions of 100-150 kb near the right end of chromosome 9 [24, 27], strongly suggesting that this region encodes another major genetic determinant of gametocytogenesis.

A genome-wide expression analysis identified a suite of genes that are activated early in gametocyte development [28] and a proteomics analysis revealed that a group of putatively exported proteins are over-represented in these stages [29]. These proteins were designated as $P$. falciparum Gametocyte EXported Proteins (PfGEXP) and the export of some of these proteins has been demonstrated, including Pfg744 [30], PfGECO [31] and PfGEXP10 [29].

Plasmodium falciparum Gametocyte EXported Protein-5 (PfGEXP5) is a member of this family. This work shows that PfGEXP5 is exported into the host cell cytoplasm from as early as $14 \mathrm{~h}$ after invasion of a sexually committed merozoite, making it the earliest gametocytespecific marker so far described. It also shows that PfGEXP5 is expressed and exported upon exposure of the F12 and GNP-A4 parasite clones to gametocyte-inducing conditions, indicating that this early gametocyte protein functions independently of the developmental switch governed by the PfAP2-G transcription factor. 


\section{Methods \\ Parasite culture}

A high gametocyte-producing P. falciparum line 3D7 [32] was cultured in human $0+\mathrm{RBCs}$, sourced from Prof G. Girelli, Dipartimento Biopatologia Umana, University of Rome 'La Sapienza' or from the Australian Red Cross Blood Service. Parasites were cultured in RPMI-HEPES supplemented with 5-10\% human serum with-without $0.25 \%$ AlbuMAX II. Transfected cultures were maintained in media supplemented with 5 nM WR99210. Parasite growth was monitored by Giemsa-stained thin blood smears. Initial synchronization of the ring stages was performed by addition of $5 \%$ sorbitol. For tight synchronization very late stage schizonts were purified by magnetic separation (CS columns; Miltenyi Biotec) [33] and mixed with uninfected RBCs and harvested $2 \mathrm{~h}$ after invasion as described previously [34]. Gametocyte cultures of the desired stage were obtained as described in [29] or using a method adapted from Fivelman et al. [35] as described in Dearnley et al. [17].

\section{Production of the PfGEXP5-GST fusion proteins and mouse immunization}

A 483 bp pfGEXP05 (PF3D7_0936600) fragment from the intron-less genomic region, encoding amino acids 104-264 of the predicted protein sequence, was cloned in a BamHI/NotI-digested pGEX-6P-3 vector and used to produce a GST fusion protein in Escherichia coli BL21 $\left(2 \mathrm{~h}\right.$ at $\left.37{ }^{\circ} \mathrm{C}\right)$, using previously described methods [36]. The protein was solubilized in $6 \mathrm{M}$ urea followed by SDSPAGE, the electro-eluted fusion protein was dialyzed and used for immunizing mice as described in [29].

\section{Production of PfGEXP5-GFP fusion protein and parasite transfection}

A full length PfGEXP5 was PCR amplified from 3D7 cDNA using the primers PfGEXP5-GFPF (agatctatgaaagatcagattgaa) and $\mathrm{R}$ (ctgcagtcccatatgtctaggtgt) and directionally cloned into the $B g l \mathrm{II}$ and PstI restriction sites (Bold) of the pCGFP-Entry vector to yield the pCGEXP5GFP-Entry vector. The CRT promoter sequence from this vector was replaced with $1500 \mathrm{bp}$ of sequence from the $5^{\prime}$ UTR of PfGEXP5. The promoter sequence from the $5^{\prime}$ UTR was PCR amplified from genomic 3D7 using the primers GEXP5-ProF (gtcgacggtggaagatcatttact) and GEXP5-ProR (agatcttctaaaaaatataaaaataaa) and was directionally cloned into the pC70GFP-Entry vector predigested with SalI and BglII to excise the CRT promoter sequence. The resultant vector contained the full length cDNA sequence and $1500 \mathrm{bp}$ of the $5^{\prime}$ UTR of the native PfGEXP5 locus. This pPfGEXP5-GFP-Entry vector was then recombined with the $\mathrm{pHH} 1$-Destination vector as per the manufacturer instructions. The resultant vector
pHH1-PfGEXP5-GFP (PfGEXP5-GFP) and transfections were performed as previously described [37].

\section{Western blot analysis}

Protein extracts from Percoll-purified gametocytes from day 2 post induction were obtained and lysed by Equinatoxin II (EqtII; [38]). Soluble and insoluble fractions were separated by SDS-PAGE, transferred to nitrocellulose filters. Primary and secondary antibodies were prepared in $3.5 \%$ skim milk in PBS and all washing steps were performed in PBS containing $0.02 \%$ Tween 20. The following primary antibodies were used: mouse anti-PfGEXP5 (1:200), mouse anti-Pfs16 (1:1000, [39]) or rabbit antiGAPDH (1:5000) [40]. Primary antibodies were added to blots and incubated overnight at $4{ }^{\circ} \mathrm{C}$. Anti-mouse and anti-rabbit antibodies $(1: 20,000)$ conjugated to horseradish peroxidase (HRP) were incubated for $1 \mathrm{~h}$ at room temperature, prior to detection by chemiluminescence.

\section{Immunofluorescence analysis}

Parasites were fixed in suspension with $4 \%$ paraformaldehyde, $0.075 \%$ glutaraldehyde and permeabilized with $0.1 \%$ Triton X-100 [41]. The antibodies used were: mouse anti-PfGEXP5 (1:100), rabbit anti-Pfg27 (1:200), mouse anti-Pfs16 (1:500) [39, 42], rabbit anti-RESA (1:200) [43], rabbit anti-KAHRP [44] and mouse/rabbit anti-GFP (1:200) (Roche) (rabbit [45]). Anti-mouse and rabbit secondary antibodies conjugated to fluorescein/Alexa 488, rhodamine/Alexa568 or Alexa 647. Images were collected using a Leica DMR fluorescence microscope fitted with a Leica cooled CCD camera, and deconvolved using the LAS V3.8.0 (Leica Application Suite 3.8.0) software, or with a Delta Vision Elite wide field deconvolution microscope. Images were processed with ImageJ [46].

\section{Results}

PfGEXP5 is expressed in early stage gametocytes and exported to the host cell cytoplasm

The two-exon gene pfgexp5 (PF3D7_0936600) encodes a protein with a predicted molecular mass of 34,909 Da. The sequence includes a predicted protein export element/host targeting signal (PEXEL/HT) motif, which indicates export to the host RBC [47], and a conserved PHIST-C domain [48]. To characterize the expression pattern and cellular location of PfGEXP5, antibodies were generated against a recombinant fragment (amino acids 104-264) fused to GST. To examine the reactivity against sexual stage parasites, a culture of gametocyte-producing 3D7 line [32] was allowed to reach a high parasitaemia to induce sexual differentiation, and $\mathrm{N}$-acetyl-glucosamine (Glc-NAc) was used to clear residual asexual parasites. The mouse antiserum was used to probe a Western blot of a sample of stage III gametocytes 
that had been treated with Equinatoxin-II (EqtII) [38] to selectively permeabilize the RBC membrane (Fig. 1a). The antiserum recognized a protein in the supernatant fraction, migrating with an apparent molecular mass of $33 \mathrm{kDa}$, which is slightly larger than the predicted size of the PEXEL-cleaved polypeptide. This is likely due to aberrant migration of some Plasmodium proteins, which is attributed to their unusual sequence composition [49, 50]. While a weakly cross-reacting band was occasionally observed in the infected RBC supernatant, no band were observed in the parasite pellet.

The above results indicate that PfGEXP5 is located in the infected RBC cytoplasm. This was directly tested using immunofluorescence microscopy of gametocytes at different stages of development from the genetically diverse parasite clones 3D7 and HB3. The samples were fixed with paraformaldehyde/glutaraldehyde, permeabilized with Triton X-100 and labelled with mouse antiPfGEXP5 and rabbit anti-Pfg27 [15]. Pfg27 is a sexual stage marker expressed in the gametocyte cytoplasm and nucleus from 30 to $36 \mathrm{~h}$ pi and maintained during further development [14]. A signal for PfGEXP5 was observed from stage I of gametocyte development in both parasite lines (Fig. 1b; Additional file 1: Figure S1A). In contrast to the Pfg27-specific signal, marking the parasite cell, the PfGEXP5-specific fluorescence is clearly detectable in the $\mathrm{RBC}$ cytoplasm in all stages analysed, thus confirming the Western blot analysis of the selectively permeabilized cells. PfGEXP5 is maintained during gametocyte development to stage IV, then declines in stage V (see Additional file 1: Figure S1B). The same analysis was conducted with an anti-GST antibody, directed against the GST moiety of the PfGEXP5 fusion protein used to immunize mice. No staining could be observed with the anti-GST antibody (see Additional file 1: Figure S1A), thus confirming the specificity of the anti-PfGEXP5 antiserum.

In the course of these experiments it was noticed that PfGEXP5 is expressed in a population of parasites that do not produce Pfg27. The parasites exhibited ring-stage morphology, with no visible haemozoin crystals. This result strongly suggests that PfGEXP5 is expressed and exported in very early (ring stage) gametocytes, before the expression of Pfg27.

\section{PfGEXP5 is initially expressed around $14 \mathrm{~h}$ post invasion} and exported to the RBC cytoplasm in parasites committed to sexual development

To investigate this point, a highly synchronous (2-h window) culture of 3D7 trophozoites was obtained. Magnet purified mature schizonts were incubated for $2 \mathrm{~h}$ with uninfected RBCs after which a sorbitol treatment was applied to obtain a culture containing only newly infected ring stages. Parasite development was followed over a 96-h period, after the first round of invasion (Fig. 2a). Immunofluorescence microscopy using antibodies against PfGEXP5 and Pfg27 showed that by $14-16 \mathrm{~h} \mathrm{pi}$, a sub-population of mononucleated parasites were positive for PfGEXP5 but negative for Pfg27, and had no detectable haemozoin pigment (Fig. 2b). By 38-40 h pi, $60 \%$ of the PfGEXP5-positive parasites were also positive for Pfg27, which identified them as stage I gametocytes (Fig. 2b). This pattern of expression was confirmed in three independent experiments.

In an independent time-point experiment, parasites sampled at 14-18 h pi were dual labelled with antibodies against PfGEXP5 and the knob-associated, histidine-rich

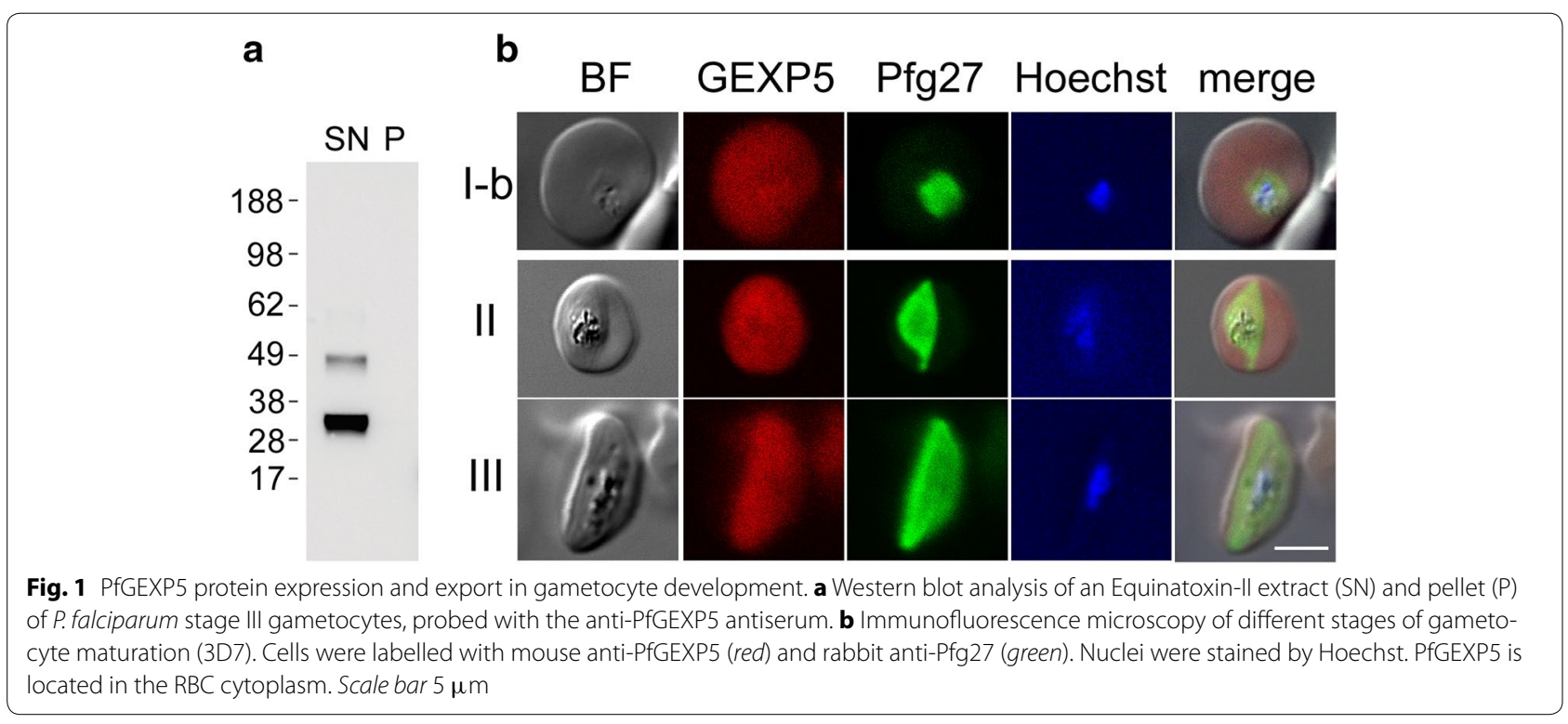




\section{a}

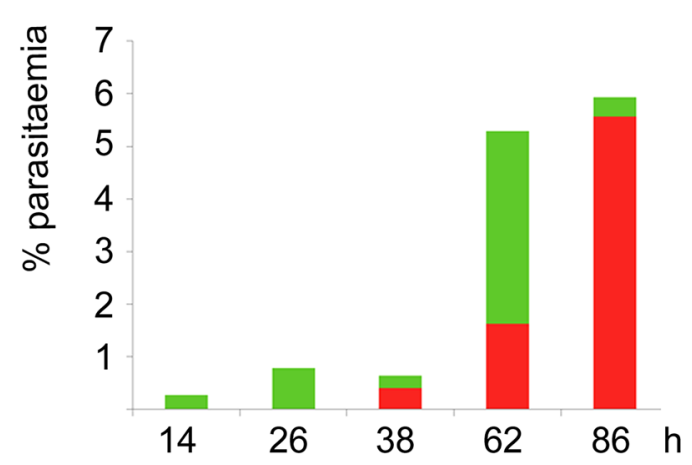

$\%$ mononucleated GEXP5+/Pfg27-

$\%$ gametocytes GEXP5+/Pfg27+

b
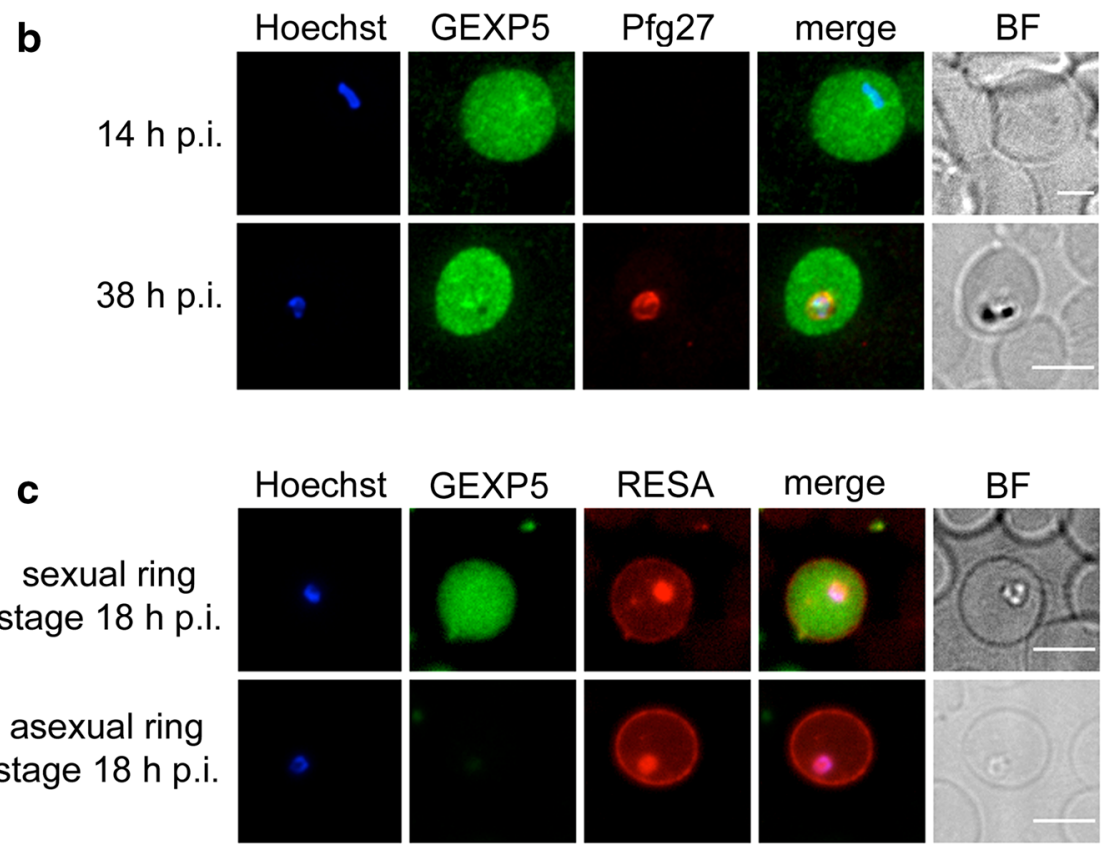

BF

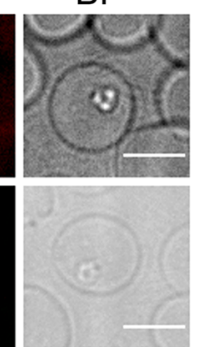

Fig. 2 Time course analysis of PfGEXP5 expression. a Time course of antigen expression after gametocyte induction in a synchronous 3D7 culture. The percentage of mononucleated parasites that are positive for PfGEXP5 alone (green) or for both PfGEXP5-positive and Pfg27 (red) are shown at different time points. These data are typical of experiments performed three times. b Representative immunofluorescence images of (top panel) a ring stage-like parasite at $14 \mathrm{~h}$ pi positive for PfGEXP5 (green) and negative for Pfg27 (red), and (lower panel) a pigmented double-positive parasite at 38 h pi. c Immunofluorescence microscopy of 18-h gametocyte and asexaul ring-infected RBCs labelled with anti-PfGEXP5 (green) and anti-RESA (red) antibodies. Brightfield images (BF) and nuclear staining are shown at right and left, respectively. Scale bar $5 \mu \mathrm{m}$

protein (KAHRP), a knob component appearing from $16 \mathrm{~h}$ pi in asexual parasites, and undetectable in the knobless stage I/early II gametocytes [51]. Failure to detect double positive parasites for such antisera shows that PfGEXP5 is not produced in asexual stages (see Additional file 1: Figure S2A). The same parasite sample at 14-18 h pi was dual labelled with antibodies against PfGEXP5 and the ring-infected erythrocyte surface antigen (RESA). RESA is one of the earliest known exported proteins in asexual parasites $[52,53]$. It associates with the spectrin network underlying the RBC membrane, where it remains detectable until $18-24 \mathrm{~h}$ pi. A population of
PfGEXP5-positive cells was observed that also exhibited RESA labelling at the RBC periphery. This was distinguishable from a population of infected RBCs where a similar RESA labelling profile was not associated with GEXP5 expression (Fig. 2c). These two cell types may represent 'sexual ring' and 'asexual ring' stages, respectively.

It was not possible to perform dual labelling of these samples with antibodies recognizing Pfs16, the earliest known gametocyte marker, due to the lack of a suitable rabbit antibody. Therefore the appearance of haemozoin was used as a surrogate marker of parasite age (postinvasion) of the above parasites. In asexual parasites, 
haemozoin is first visible at about $14 \mathrm{~h}$ pi [54]. Most (79 \%) of the PfGEXP5-positive parasites exhibited no visible haemozoin crystals (e.g., Fig. 2b, c), indicating that haemoglobin digestion was minimal, while small haemozoin crystals were apparent in the other $21 \%$.

These experiments confirm that the anti-PfGEXP5 antiserum is able to recognize sexually committed parasites from as early as $14 \mathrm{~h}$ pi and provides the first evidence that RESA is expressed in early gametocytes.

\section{A PfGEXP5-GFP chimera is exported to the RBC cytoplasm}

To investigate PfGEXP5 expression and export in an independent approach, a $P$. falciparum transfectant line was generated expressing full-length PfGEXP5 C-terminally tagged with green fluorescent protein (GFP), autologously regulated by $1.5 \mathrm{~kb}$ of the pfgexp 5 genomic upstream region. The parent (3D7) and transfectant (PfGEXP5-GFP) lines were induced to undergo sexual differentiation and stage II-III gametocytes were harvested and selectively lysed with Eqt-II to release the host cell content. Anti-PfGEXP5 antibodies recognized the endogenous protein in the Eqt-II supernatant and an additional band ( $\sim 60 \mathrm{kDa})$ corresponding to the PfGEXP5-GFP (Fig. 3a). The relative intensity of the upper band indicates that PfGEXP5-GFP is expressed at a similar level to the endogenous PfGEXP5. Some of the PfGEXP5-GFP chimera is still detectable in the parasite fraction, indicating a less efficient export of the chimera beyond the parasitophorous vacuole (PV), which is not breached by Eqt-II, compared to that of the endogenous protein. When PfGEXP5-GFP transfectants were probed with anti-GFP, only the $62 \mathrm{kDa}$ protein species was detected, with no reactivity in the 3D7 parent (Fig. 3a).

Live cell imaging of the PfGEXP5-GFP transfectant parasites provided further evidence for export of the protein to the host RBC compartment, with the PV as an intermediate compartment (Fig. 3b). In the stage IV and $\mathrm{V}$ gametocytes the host cytoplasm is reduced to a thin layer around the parasite, with PfGEXP5-GFP concentrated in this compartment. The persistence of the fluorescence signal in stage $\mathrm{V}$ gametocytes may be due to the high stability of the GFP tag.

Live imaging of asexual parasites grown at high parasitaemia to induce gametocytogenesis showed that GFP fluorescence was not detectable in multinucleated parasites, indicating that PfGEXP5 is not expressed in sexually committed schizonts (see Additional file 1: Figure S2B).

Immunofluorescence microscopy confirmed that PfGEXP5-GFP is expressed and exported to the host cell cytoplasm in Pfs16-positive cells (Fig. 3c). In the earliest stages post-induction some cells were observed that were positive for PfGEXP5-GFP but negative for Pfs16
(Fig. 3c), confirming that expression of PfGEXP5 precedes that of Pfs16. No Pfs16-positive/PfGEXP5-GFPnegative parasites were observed, indicating that all stage I gametocytes express PfGEXP5. Consistent with this, the PfGEXP5-GFP positive cells did not exhibit KAHRP labelling at the RBC surface, while KAHRP-positive cells (i.e., asexual stages) were negative for exported PfGEXP5-GFP (Fig. 3d).

These results show that the $1.5 \mathrm{~kb}$ of genomic upstream region of the $p f g \exp 5$ gene is sufficient to confer a gametocyte-specific pattern of expression to the PfGEXP5GFP fusion, and that it contains the pfgexp 5 promoter. The data further indicate that PfGEXP5 is the earliest sexual stage-marker so far described. They also confirm that PfGEXP5 is only expressed in post-invasion sexually committed parasites and is not present at detectable levels in the committed schizonts from the previous cycle.

\section{PfGEXP5 expression is not under the control of the master} regulator of Plasmodium sexual differentiation AP2G

The PfGEXP5 marker was exploited to investigate early events in parasite development in the gametocyte defective clones $\mathrm{F} 12$ and GNP-A4, in which the mutated PfAP2-G master regulator of sexual differentiation is unable to trigger the expression of 12 early gametocyte transcripts, including those from genes $p f s 16$ and $p f g 27$, and to produce gametocytes [25].

Synchronous F12, GNP-A4 or parental 3D7 parasites were treated using standard gametocyte-inducing conditions. At $12-16 \mathrm{~h}$ pi immunofluorescence analysis revealed a sub-population of parasites in each of the three lines that was positive for PfGEXP5 in the host RBC cytoplasm (Fig. 4a). The percentage of PfGEXP5positive parasites/total parasites $(\sim 15 \%)$ was similar in 3D7 and in the two independent AP2G-defective lines (Fig. 4b). Immunofluorescence analysis using anti-Pfg27 at $36 \mathrm{~h}$ pi showed that, as expected, no parasite populations became positive for the above early gametocyte marker in F12 and in GNP-A4. In contrast, anti-Pfg27positive stage I gametocytes were observed in the 3D7 line as expected. At $30 \mathrm{~h}$ pi, parasite samples from the F12 and 3D7 cultures were collected, treated with EqtII to release the soluble content of the host RBC and analysed by Western blot. This experiment confirmed that PfGEXP5 was present at comparable levels in the RBC soluble fraction of both parasites (Fig. 4c).

The observation that expression and export of PfGEXP5 in parasites at $14 \mathrm{~h}$ pi are comparable irrespective of the presence of a functional AP2G indicates that PfGEXP5 expression is not under control of this regulator. The transcriptional profiles of pfgexp5 in wild type and in the AP2G-defective F12 parasite line are similar in the period 3-45 h pi (Additional file 1: Figure S3); kindly 


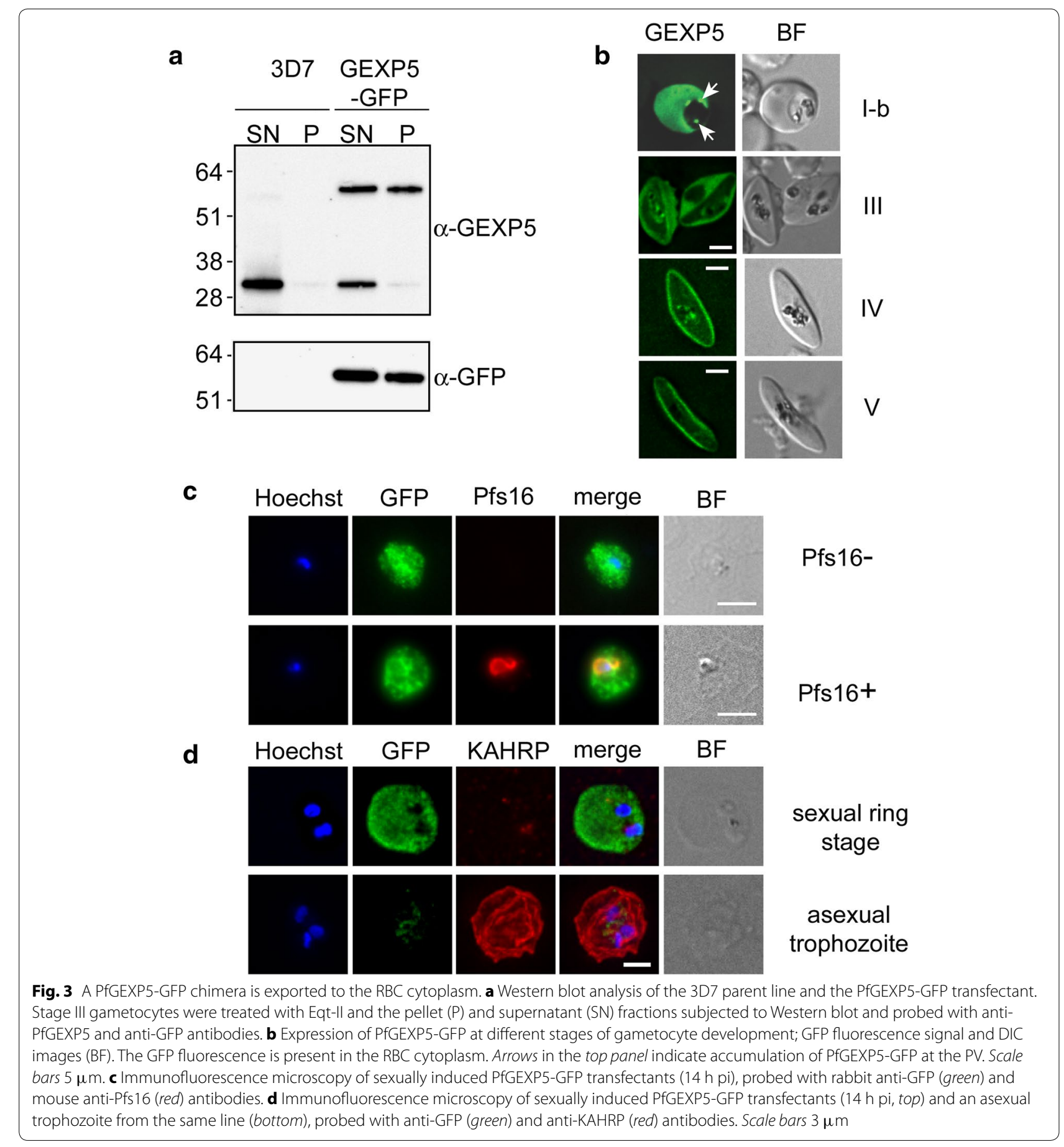

provided by Manuel Llinas, University of Pennsylvania, USA, from published data [25]). This is consistent with the observation that the PfAP2-G binding site consensus sequence $\mathrm{G}(\mathrm{T}, \mathrm{A}, \mathrm{C}) \mathrm{GTAC}$ is not present in over $4 \mathrm{~kb}$ of the upstream region of the $p f g \exp 5$ gene. These data also indicate that in AP2-G-defective parasites sexual commitment is initiated and then aborted.

\section{Discussion}

PfGEXP5 is the earliest available marker of Plasmodium falciparum gametocyte development

In this work evidence is provided that PfGEXP5 is expressed from $14 \mathrm{~h}$ pi in mononucleated, haemozoinfree, ring stage-like parasites that subsequently produce Pfg27. Thus reagents against PfGEXP5 represent 


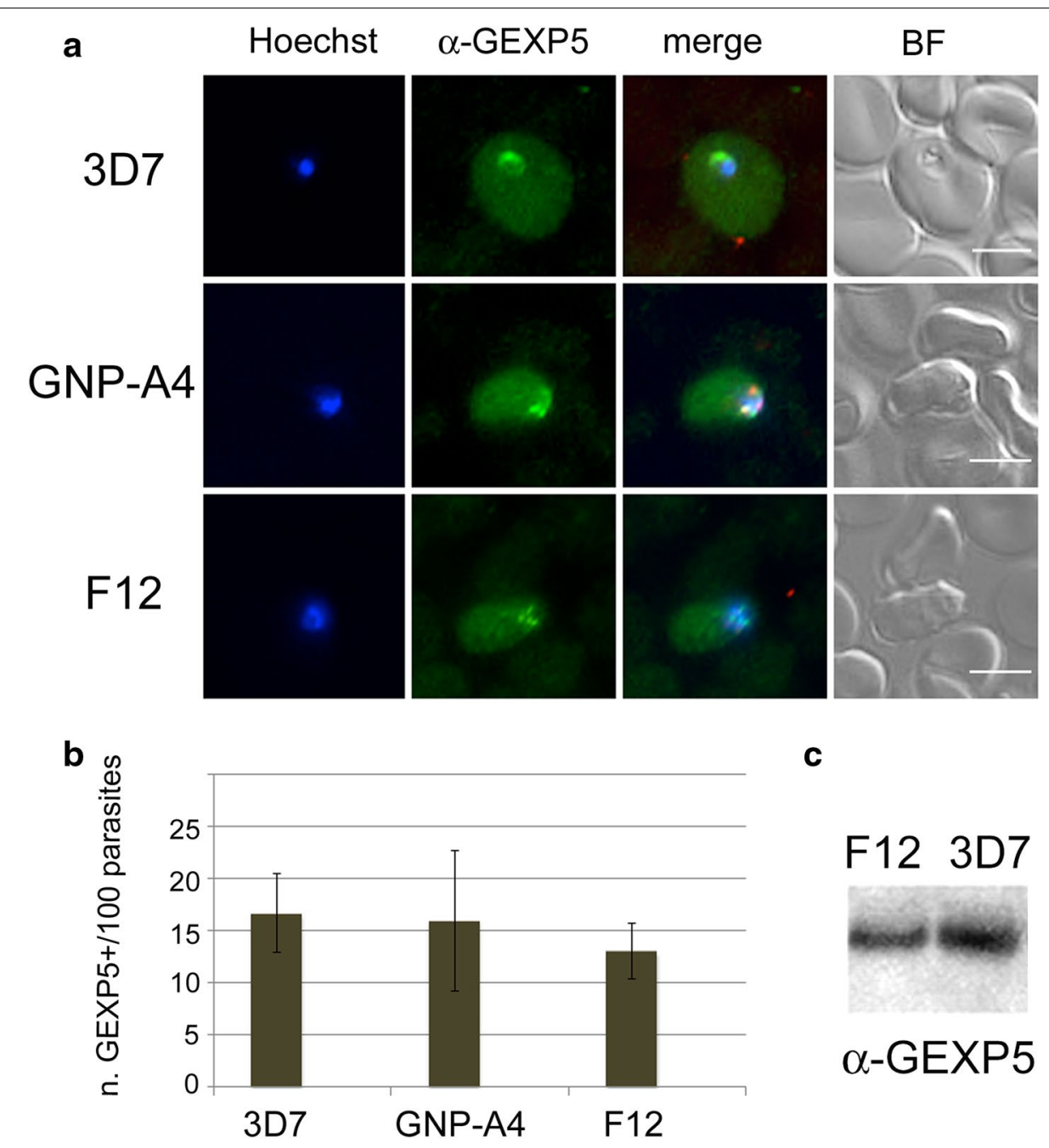

Fig. 4 PfGEXP5 expression and export in AP2-G defective parasites. a Immunofluorescence microscopy of sexually induced GPN-A4 and F12 parasites at $14 \mathrm{~h}$ pi, probed with anti-PfGEXP5 antibodies. Scale bar $5 \mathrm{~mm}$. b Percent of the above PfGEXP5 positive parasites over total parasites counted at $14 \mathrm{~h}$ pi. Error bars are standard deviation (from two separate experiments). c Western blot analysis with anti-PfGEXP5 antibodies on samples of gametocyte-induced cultures of F12 and 3D7 parasites at $30 \mathrm{~h}$ pi

potentially valuable tools to investigate very early events in P. falciparum sexual differentiation.

Gametocytogenesis is of significant interest as it is the only developmental branch point in the P. falciparum life cycle. Gametocyte conversion rates can be modulated in vitro by changing the asexual parasitaemia [5] or otherwise stressing the culture $[55,56]$, suggesting that the parasite can respond to environmental cues. However an alternative proposal [8] is that a fixed fraction of asexual schizonts commit to gametocytogenesis at every round of asexual replication. As it is virtually impossible to address these questions in the analysis of gametocyte conversion rates in $P$. falciparum natural infections, the availability of a ring stage gametocyte marker will clearly be an invaluable tool.
Several studies have described genes and proteins of possible interest as early gametocyte markers. Transgenic chimeras for several genes expressed in gametocytogenesis have been detected in sub-populations of schizonts, which are assumed to be committed to sexual differentiation. These are pfg14.748 [30], pfSET [57], pfg27 [36], pfnek4 [58], pfgdv1 [8], and pfAP2-G [25]. However it is not yet clear that all the merozoites expressing these markers develop as gametocytes and continue to produce these proteins very early upon re-invasion. This limits their usefulness as very early sexual markers. Other proteins, such as Pfmdv-1/peg3 and PfPeg4, have been described to appear in early gametocytes, but not in putatively sexually committed schizonts. However, these are expressed at the same time or shortly after 
Pfs16, Pfg27 [28] and PfGECO [31]. PfGEXP5 is the only protein so far described to be expressed in mononucleated, ring stage-like very early gametocytes from as early as $14 \mathrm{~h}$ pi. As the morphology of the PfGEXP5-positive ring stages is indistinguishable from that of asexual ring stages, it is likely that reagents against PfGEXP5 will detect very early gametocytes still in circulation before homing to their sequestration sites. This will provide a breakthrough tool for studies of gametocyte population dynamics in natural infections. It will also enable monitoring of the human infectious reservoirs and will facilitate assessment of the effects of anti-malarial drugs on gametocyte carriage.

\section{PfGEXP5 expression and host cell remodelling at the onset of gametocytogenesis}

The export of the PfGEXP5 and PfGEXP5-GFP into the host cytoplasm was observed, presumably mediated by the PEXEL/HT motif. This is consistent with previous studies showing host cell remodelling in the early phases of gametocyte maturation [29]. Functional roles of exported gametocyte proteins are being elucidated. Disruption of genes PFE1615c and MAL7P1.171, encoding PEXEL/HT-containing proteins, result respectively in failure to produce gametocytes and failure to progress beyond stage I of gametocytogenesis [59]. This work shows that the timing of expression of PfGEXP5 overlaps with that of the cytoskeleton-interacting parasite protein RESA, with the RESA signal waning as the PfGEXP5 signal increases. This provides the first evidence that RESA is expressed in early stage gametocytes, where it may interact with gametocyte-specific proteins. RESA is expressed in the final stages of schizont development, stored in apical organelles within the individual merozoites [60], then transported to the RBC membrane shortly after invasion [61]. Thus its expression may be controlled separately from that of early gametocyte genes.

The $p f g \exp 5$ gene encodes a protein with a conserved PHIST domain, recently classified as belonging to the PHIST subgroup C [48]. Other PHIST C and PHIST B proteins are expressed maximally during schizogony and after invasion in ring stage parasites [62]. The conserved PHIST C domain (a six-helical, segment-structural element that is thought to be involved in protein-protein interactions) may give a clue to its function. PHIST-containing proteins are found only in the genus Plasmodium suggesting that the domain evolved after the divergence of the lineage from other Apicomplexa and that the protein family has undergone a dramatic expansion in $P$. falciparum [48, 62]. A similar domain is found in RESA where it may play a role in binding to spectrin and protecting the RBC membrane from damage during shear stress and thermal challenge $[63,64]$. Another member of the PHIST family (PF3D7_0402000) binds to protein 4.1 , an interaction that appears to lead to dissociation of a sub-population of protein 4.1 molecules from the cytoskeleton and relocation to the PV membrane [65]. These observations lead us to speculate that PfGEXP5 plays a role in re-sculpting the host RBC cytoplasm from a very early stage of gametocyte differentiation.

\section{PfGEXP5 expression regulation suggests the existence of multiple checkpoints governing Plasmodium falciparum sexual differentiation}

The genes that control gametocyte development are beginning to be characterized. Recently, a putative transcription factor, PfAP2-G, was identified as a master regulator controlling the switch to sexual stage development. This was based on the observations that PfAP2-G positively regulates the expression of a subset of early gametocyte genes, including $p f s 16$ and $p f g 27 / 25$, and that PfAP2-G-defective parasites fail to produce gametocytes. The observation that such mutants have a selective growth advantage over the wild-type parasites was interpreted as a demonstration that no sexually committed schizonts are produced $[25,66]$.

Surprisingly, PfGEXP5 is expressed and successfully exported in two clones (F12 and GNP-A4) that are defective for PfAP2-G. This is supported by data from transcriptional analyses of PfAP2- $\mathrm{G}^{+}$and PfAP2 $-\mathrm{G}^{-}$parasites indicating that the $p f g \exp 5$ transcript is not upregulated by PfAP2-G at the onset of sexual differentiation [25]. These observations indicate that regulation of $p f g \exp 5$ is most likely governed by a PfAP2-G independent regulatory mechanism.

The fact that sub-populations of F12 and GNP-A4 parasites are able to express and export PfGEXP5 under conditions that normally trigger gametocytogenesis indicates that commitment to sexual development can take place in the absence of functional PfAP2-G. However PfGEXP5 expression is not sufficient to support continued gametocyte development in a background where PfAP2-G is mutated (and thus other early sexual-stage transcripts are not up-regulated). This suggests that multiple mechanisms cooperate to support sexual differentiation. $p f g$ exp5 expression may act in synergy with the PfAP2-G regulatory cascade, perhaps as an additional checkpoint in commitment or in the earliest steps of gametocyte differentiation.

\section{Conclusions}

The expression and export of PfGEXP5 represent the earliest post-invasion sexual stage marker described to date. This opens the possibility to generate tools able to identify sexually committed ring-stage parasites in natural infections. These could be antibody-based and 
developed as a rapid diagnostic test or used for immunofluorescence analysis of smears collected from patients. Alternatively, levels of pfGEXP5 transcript could be monitored using quantitative real time (qRT-PCR), possibly revealing freely circulating early sexual stages. In addition, this early gametocyte marker will facilitate efforts to identify and map the human malaria transmission reservoir in human populations. Finally, the finding that regulation of PfGEXP5 does not depend on the AP2-G master regulator of parasite sexual development suggests that, after sexual commitment, differentiation progresses through multiple checkpoints in the early phase of gametocytogenesis.

\section{Additional file}

Additional file 1 Figure S1. Immunofluorescence analysis of 3D7 and HB3 gametocytes with antibodies against PfGEXP5, Pfg27 and GST. Figure S2. Analysis of the production of endogenous or GFP-fused PFGEXP5 in asexual stages. Figure $\mathbf{S 3}$. pfgexp 5 transcriptional profile in PfAP2G- and PfAP2G + parasites.

\begin{abstract}
Abbreviations
GEXP5: Gametocyte EXported Protein-5; RBC: red blood cell; KAHRP: knobassociated, histidine-rich protein; RESA: ring-infected erythrocyte surface antigen; PV: parasitophorous vacuole; GFP: green fluorescent protein; Eqtll: equinatoxin-II; Glc-NAc: N-acetyl-glucosamine.
\end{abstract}

\section{Authors' contributions}

MT purified the PfGEXP5 recombinant protein for mouse immunization and characterized the antibody, performed the time course and immunofluorescence analyses in 3D7, F12 and GNP-A4 P. falciparum lines; MWAD produced the PfGEXP5-GFP transgenic parasites, performed immunofluorescence and Western blot experiments; OL performed immunofluorescence experiments; SYY produced the PfGEXP5-GST expression plasmid and bacterial extracts; LT conceived and analysed experiments and contributed to the manuscript; PA conceived and analysed experiments and contributed to the manuscript. All authors read and approved the final manuscript.

\section{Author details}

${ }^{1}$ Dipartimento di Malattie Infettive, Parassitarie e Immunomediate, Istituto Superiore di Sanità, Viale Regina Elena 299, 00161 Rome, Italy. ${ }^{2}$ Department of Biochemistry and Molecular Biology, Bio21 Molecular Science and Biotechnology Institute, The University of Melbourne, Melbourne, VIC, Australia. ${ }^{3}$ Present Address: The Francis Crick Institute, Mill Hill Laboratory, The Ridgeway, Mill Hill, London NW7 1AA, UK. ${ }^{4}$ Present Address: Department of Parasitology, Biomedical Primate Research Centre, PO Box 306, 2280 GH Rijswijk, The Netherlands.

\section{Acknowledgements}

We thank Dr. Don Gardiner and Dr. Katharine Trenholme (QIMR Berghofer Medical Research Institute) for support in the production of the $\mathrm{pHH} 1-\mathrm{PfG}$ EXP5-GFP plasmid; Dr. David Baker (London School of Hygiene and Tropical Medicine) for providing the GNP-A4 parasite line, Prof Robin Anders (La Trobe University), Prof Mike Ryan (Monash University) and Prof Alan Cowman (Walter and Eliza Hall Institute) for generously providing antibodies, Prof G. Girelli, Dipartimento di Biopatologia Umana, University of Rome "La Sapienza' for providing blood samples for parasite culture at ISS, Rome; Mr Leonardo Picci for mouse immunization and Ms Shannon Kenny for technical assistance. This work was supported by the EU FP7 Grant n.242095 EVIMalaR, by a travel fellowship to MLT from the FP7 Marie Curie ISRES Grant n.269238 OzMalNet, the National Health and Medical Research Council of Australia, the Australia Research Council and Grant OPP1040394 of the Global Health Program of the
Bill and Melinda Gates Foundation. MWAD was supported by an NHMRC Early career Fellowship and LT is an ARC Australian Professorial Fellow.

\section{Compliance with ethical guidelines}

\section{Competing interests}

The authors declare that they have no competing interests.

Received: 24 June 2015 Accepted: 16 August 2015

Published online: 28 August 2015

\section{References}

1. WHO. World malaria report, 2014. Geneva: World_Health_Organization; 2014.

2. Breman JG, Alilio MS, Mills A. Conquering the intolerable burden of malaria: what's new, what's needed: a summary. Am J Trop Med Hyg. 2004; $71: 1-15$

3. White NJ, Pukrittayakamee S, Hien TT, Faiz MA, Mokuolu OA, Dondorp AM. Malaria. Lancet. 2013;383:723-35.

4. Alano P. Plasmodium falciparum gametocytes: still many secrets of a hidden life. Mol Microbiol. 2007;66:291-302.

5. Bruce MC, Alano P, Duthie S, Carter R. Commitment of the malaria parasite Plasmodium falciparum to sexual and asexual development. Parasitology. 1990;100:191-200.

6. Silvestrini F, Alano P, Williams JL. Commitment to the production of male and female gametocytes in the human malaria parasite Plasmodium falciparum. Parasitology. 2000;121:465-71.

7. Smith TG, Lourenco P, Carter R, Walliker D, Ranford-Cartwright LC. Commitment to sexual differentiation in the human malaria parasite, Plasmodium falciparum. Parasitology. 2000;121:127-33.

8. Eksi S, Morahan BJ, Haile Y, Furuya T, Jiang H, Ali O, et al. Plasmodium falciparum gametocyte development 1 (Pfgdv1) and gametocytogenesis early gene identification and commitment to sexual development. PLoS Pathog. 2012;8:e1002964.

9. Hawking F, Wilson ME, Gammage K. Evidence for cyclic development and short-lived maturity in the gametocytes of Plasmodium falciparum. Trans R Soc Trop Med Hyg. 1971;65:549-59.

10. Dixon MW, Dearnley MK, Hanssen E, Gilberger T, Tilley L. Shape-shifting gametocytes: how and why does P. falciparum go banana-shaped? Trends Parasitol. 2012;28:471-8.

11. Bruce MC, Carter RN, Nakamura K, Aikawa M, Carter R. Cellular location and temporal expression of the Plasmodium falciparum sexual stage antigen Pfs16. Mol Biochem Parasitol. 1994;65:11-22.

12. Baker DA, Daramola O, McCrossan MV, Harmer J, Targett GA. Subcellular localization of Pfs 16, a Plasmodium falciparum gametocyte antigen. Parasitology. 1994;108:129-37.

13. Sharma A, Sharma I, Kogkasuriyachai D, Kumar N. Structure of a gametocyte protein essential for sexual development in Plasmodium falciparum. Nat Struct Biol. 2003;10:197-203.

14. Camarda G, Bertuccini L, Singh SK, Salzano AM, Lanfrancotti A, Olivieri A, et al. Regulated oligomerisation and molecular interactions of the early gametocyte protein Pfg27 in Plasmodium falciparum sexual differentiation. Int J Parasitol. 2010;40:663-73.

15. Alano P, Premawansa S, Bruce MC, Carter R. A stage specific gene expressed at the onset of gametocytogenesis in Plasmodium falciparum. Mol Biochem Parasitol. 1991;46:81-8.

16. Sinden RE. Gametocytogenesis of Plasmodium falciparum in vitro: an electron microscopic study. Parasitology. 1982;84:1-11.

17. Dearnley MK, Yeoman JA, Hanssen E, Kenny S, Turnbull L, Whitchurch CB, et al. Origin, composition, organization and function of the inner membrane complex of Plasmodium falciparum gametocytes. J Cell Sci. 2012;125:2053-63.

18. Joice R, Nilsson SK, Montgomery J, Dankwa S, Egan E, Morahan B, et al. Plasmodium falciparum transmission stages accumulate in the human bone marrow. Sci Transl Med. 2014;6:244re245.

19. Aguilar R, Magallon-Tejada A, Achtman AH, Moraleda C, Joice R, Cistero $P$, et al. Molecular evidence for the localization of Plasmodium falciparum immature gametocytes in bone marrow. Blood. 2014;123:959-66. 
20. Baker DA. Malaria gametocytogenesis. Mol Biochem Parasitol. 2010;172:57-65

21. Pelle KG, Oh K, Buchholz K, Narasimhan V, Joice R, Milner DA, et al. Transcriptional profiling defines dynamics of parasite tissue sequestration during malaria infection. Genome Med. 2015;7:19.

22. Makanga M. A review of the effects of artemether-lumefantrine on gametocyte carriage and disease transmission. Malar J. 2014;13:291.

23. WWARN. The effect of dose on the antimalarial efficacy of artemetherlumefantrine: a systematic review and pooled analysis of individual patient data. Lancet Infect Dis. 2015;15:692-702.

24. Alano P, Roca L, Smith D, Read D, Carter R, Day K. Plasmodium falciparum: parasites defective in early stages of gametocytogenesis. Exp Parasitol. 1995;81:227-35.

25. Kafsack BF, Rovira-Graells N, Clark TG, Bancells C, Crowley VM, Campino SG, et al. A transcriptional switch underlies commitment to sexual development in malaria parasites. Nature. 2014;507:248-52.

26. Shirley MW, Biggs BA, Forsyth KP, Brown HJ, Thompson JK, Brown GV, et al. Chromosome 9 from independent clones and isolates of Plasmodium falciparum undergoes subtelomeric deletions with similar breakpoints in vitro. Mol Biochem Parasitol. 1990;40:137-45.

27. Day KP, Karamalis F, Thompson J, Barnes DA, Peterson C, Brown H, et al. Genes necessary for expression of a virulence determinant and for transmission of Plasmodium falciparum are located on a 0.3-megabase region of chromosome 9. Proc Natl Acad Sci USA. 1993:90:8292-6.

28. Silvestrini F, Bozdech Z, Lanfrancotti A, Di Giulio E, Bultrini E, Picci L, et al. Genome-wide identification of genes upregulated at the onset of gametocytogenesis in Plasmodium falciparum. Mol Biochem Parasitol. 2005;143:100-10.

29. Silvestrini F, Lasonder E, Olivieri A, Camarda G, van Schaijk B, Sanchez M, et al. Protein export marks the early phase of gametocytogenesis of the human malaria parasite Plasmodium falciparum. Mol Cell Proteomics. 2010;9:1437-48.

30. Eksi S, Haile Y, Furuya T, Ma L, Su X, Williamson KC. Identification of a subtelomeric gene family expressed during the asexual-sexual stage transition in Plasmodium falciparum. Mol Biochem Parasitol. 2005;143:90-9.

31. Morahan BJ, Strobel C, Hasan U, Czesny B, Mantel PY, Marti M, et al. Functional analysis of the exported type IV HSP40 protein PfGECO in Plasmodium falciparum gametocytes. Eukaryot Cell. 2011;10:1492-503.

32. Walliker D. Genes, vaccines and variation in malaria. Parasitol Today. 1986:2:43-4.

33. Silvestrini F, Tiburcio M, Bertuccini L, Alano P. Differential adhesive properties of sequestered asexual and sexual stages of Plasmodium falciparum on human endothelial cells are tissue independent. PLoS One. 2012;7:e31567.

34. Xie SC, Dogovski C, Kenny S, Tilley L, Klonis N. Optimal assay design for determining the in vitro sensitivity of ring stage Plasmodium falciparum to artemisinins. Int J Parasitol. 2014;44:893-9.

35. Fivelman QL, McRobert L, Sharp S, Taylor CJ, Saeed M, Swales CA, et al. Improved synchronous production of Plasmodium falciparum gametocytes in vitro. Mol Biochem Parasitol. 2007;154:119-23.

36. Olivieri A, Silvestrini F, Sanchez M, Alano P. A 140-bp AT-rich sequence mediates positive and negative transcriptional control of a Plasmodium falciparum developmentally regulated promoter. Int J Parasitol. 2008;38:299-312.

37. Deitsch K, Driskill C, Wellems T. Transformation of malaria parasites by the spontaneous uptake and expression of DNA from human erythrocytes. Nucleic Acids Res. 2001;29:850-3.

38. Jackson KE, Spielmann T, Hanssen E, Adisa A, Separovic F, Dixon MW, et al. Selective permeabilization of the host cell membrane of Plasmodium falciparum-infected red blood cells with streptolysin $\mathrm{O}$ and equinatoxin II. Biochem J. 2007;403:167-75.

39. Dixon MWA, Peatey CL, Gardiner DL, Trenholme KR. A green fluorescent protein based assay for determining gameotycte production in Plasmodium falciparum. Mol Biochem Parasitol. 2009;163:123-6.

40. Satchell JF, Malby RL, Luo CS, Adisa A, Alpyurek AE, Klonis N, et al. Structure of glyceraldehyde-3-phosphate dehydrogenase from Plasmodium falciparum. Acta Crystallogr D Biol Crystallogr. 2005;61:1213-21.

41. Tonkin CJ, van Dooren GG, Spurck TP, Struck NS, Good RT, Handman E, et al. Localization of organellar proteins in Plasmodium falciparum using a novel set of transfection vectors and a new immunofluorescence fixation method. Mol Biochem Parasitol. 2004;137:13-21.
42. Baker DA, Thompson J, Daramola OO, Carlton JMR, Targett GAT. Sexual stage specific RNA expression of a new Plasmodium falciparum gene detected by in situ hybridization. Mol Biochem Parasitol. 1995;72:193-201.

43. Collins WE, Anders RF, Pappaioanou M, Campbell GH, Brown GV, Kemp DJ, et al. Immunization of Aotus monkeys with recombinant proteins of an erythrocyte surface antigen of Plasmodium falciparum. Nature. 1986;323:259-62.

44. Waterkeyn JG, Wickham ME, Davern KM, Cooke BM, Coppel RL, Reeder JC, et al. Targeted mutagenesis of Plasmodium falciparum erythrocyte membrane protein 3 (PfEMP3) disrupts cytoadherence of malaria-infected red blood cells. EMBO J. 2000;19:2813-23.

45. Humphries AD, Streimann IC, Stojanovski D, Johnston AJ, Yano M, Hoogenraad NJ, et al. Dissection of the mitochondrial import and assembly pathway for human Tom40. J Biol Chem. 2005;280:11535-43.

46. Schneider CA, Rasband WS, Eliceiri KW. NIH Image to ImageJ: 25 years of image analysis. Nat Methods. 2012;9:671-75.

47. Marti M, Good RT, Rug M, Knuepfer E, Cowman AF. Targeting malaria virulence and remodeling proteins to the host erythrocyte. Science. 2004;306:1930-3.

48. Frech C, Chen N. Variant surface antigens of malaria parasites: functional and evolutionary insights from comparative gene family classification and analysis. BMC Genom. 2013;14:427.

49. Spycher C, Klonis N, Spielmann T, Kump E, Steiger S, Tilley L, et al. MAHRP-1, a novel Plasmodium falciparum histidine-rich protein, binds ferriprotoporphyrin IX and localizes to the Maurer's clefts. J Biol Chem. 2003;278:35373-83.

50. Hanssen E, Hawthorne P, Dixon MW, Trenholme KR, McMillan PJ, Spielmann T, Gardiner DL, Tilley L. Targeted mutagenesis of the ring-exported protein-1 of Plasmodium falciparum disrupts the architecture of Maurer's cleft organelles. Mol Microbiol. 2008;69:938-53.

51. Tiburcio M, Silvestrini F, Bertuccini L, Sander AF, Turner L, Lavstsen T, et al. Early gametocytes of the malaria parasite Plasmodium falciparum specifically remodel the adhesive properties of infected erythrocyte surface. Cell Microbiol. 2012;15:647-59.

52. Foley M, Tilley $L$, Sawyer WH, Anders RF. The ring-infected erythrocyte surface antigen of Plasmodium falciparum associates with spectrin in the erythrocyte membrane. Mol Biochem Parasitol. 1991;46:137-47.

53. Coppel RL, Lustigman S, Murray L, Anders RF. MESA is a Plasmodium falciparum phosphoprotein associated with the erythrocyte membrane skeleton. Mol Biochem Parasitol. 1988;31:223-31.

54. Abu Bakar NA, Klonis N, Hanssen E, Chan C, Tilley L. Digestive-vacuole genesis and endocytic processes in the early intraerythrocytic stages of Plasmodium falciparum. J Cell Sci. 2010;123:441-50.

55. Williams JL. Stimulation of Plasmodium falciparum gametocytogenesis by conditioned medium from parasite cultures. Am J Trop Med Hyg. 1999;60:7-13.

56. Dyer M, Day KP. Regulation of the rate of asexual growth and commitment to sexual development by diffusible factors from in vitro cultures of Plasmodium falciparum. Am J Trop Med Hyg. 2003;68:403-9.

57. Pace T, Olivieri A, Sanchez M, Albanesi V, Picci L, Siden Kiamos I, et al. Set regulation in asexual and sexual Plasmodium parasites reveals a novel mechanism of stage-specific expression. Mol Microbiol. 2006;60:870-82.

58. Reininger L, Garcia M, Tomlins A, Muller S, Doerig C. The Plasmodium falciparum, Nima-related kinase Pfnek-4: a marker for asexual parasites committed to sexual differentiation. Malar J. 2012;11:250.

59. Ikadai H, Shaw Saliba K, Kanzok SM, McLean KJ, Tanaka TQ, Cao J, et al. Transposon mutagenesis identifies genes essential for Plasmodium falciparum gametocytogenesis. Proc Natl Acad Sci USA. 2013;110:E1676-84.

60. Aikawa M, Torii M, Sjolander A, Berzins K, Perlmann P, Miller LH. Pf155/ RESA antigen is localized in dense granules of Plasmodium falciparum merozoites. Exp Parasitol. 1990;71:326-9.

61. Culvenor JG, Day KP, Anders RF. Plasmodium falciparum ring-infected erythrocyte surface antigen is released from merozoite dense granules after erythrocyte invasion. Infect Immun. 1991;59:1183-7.

62. Sargeant TJ, Marti M, Caler E, Carlton JM, Simpson K, Speed TP, Cowman AF. Lineage-specific expansion of proteins exported to erythrocytes in malaria parasites. Genome Biol. 2006;7:R12.

63. Da Silva E, Foley M, Dluzewski AR, Murray $L$, Anders RF, Tilley L. The Plasmodium falciparum protein RESA interacts with the erythrocyte cytoskeleton and modifies erythrocyte thermal stability. Mol Biochem Parasitol. 1994;66:59-69. 
64. Pei X, Guo X, Coppel R, Bhattacharjee S, Haldar K, Gratzer W, et al. The ring-infected erythrocyte surface antigen (RESA) of Plasmodium falciparum stabilizes spectrin tetramers and suppresses further invasion. Blood. 2007:110:1036-42.

65. Parish LA, Mai DW, Jones ML, Kitson EL, Rayner JC. A member of the Plasmodium falciparum PHIST family binds to the erythrocyte cytoskeleton component band 4.1. Malar J. 2013;12:160.
66. Sinha A, Hughes KR, Modrzynska KK, Otto TD, Pfander C, Dickens NJ, et al. A cascade of DNA-binding proteins for sexual commitment and development in Plasmodium. Nature. 2014;507:253-7.

\section{Submit your next manuscript to BioMed Central} and take full advantage of:

- Convenient online submission

- Thorough peer review

- No space constraints or color figure charges

- Immediate publication on acceptance

- Inclusion in PubMed, CAS, Scopus and Google Scholar

- Research which is freely available for redistribution

Submit your manuscript at

www.biomedcentral.com/submit

() Biomed Central 